\title{
OFDM WITH TRAILING ZEROS VERSUS OFDM WITH CYCLIC PREFIX: LINKS, COMPARISONS AND APPLICATION TO THE HIPERLAN/2 SYSTEM
}

\author{
Bertrand Muquet $^{1}$, Marc de Courville ${ }^{1}$, Pierre Duhamel ${ }^{2}$ and Georgios Giannakis ${ }^{3}$ \\ ${ }^{1}$ Centre de Recherche Motorola Paris, Espace Technologique Saint-Aubin 91193 Gif-sur-Yvette, France - email: courvill@crm.mot.com \\ ${ }^{2}$ École nationale Supérieure des Télécommunications, 46 rue Barrault 75013 Paris, France - email: duhameletsi.enst.fr \\ ${ }^{3}$ Dept. of ECE, Univ. of Minnesota, 200 Union Street SE, Minneapolis MN 55455; US - email: georgios@ece.umn.edu
}

\begin{abstract}
This paper proposes a simple equalizer for a recent multicarrier block transmission scheme which pads zeros (as opposed to a cyclic prefix) in each transmitted block. In the absence of high-power amplifier induced nonlinear effects, it is shown that the resulting scheme is simply the dual of the classical Cyclic Prefix OFDM transceiver. Comparison between the two systems that takes into account nonlinear distortions introduced by the clipper is also performed in the practical context of the wireless broadband $5 \mathrm{GHz}$ HiperLAN/2 system. Both the classical pilot-based as well as novel (semi-) blind subspace algorithms are applied for channel estimation. The advantage of subspace methods in lowering the variation of channel tracking is corroborated with simulations which verify practically accurate channel estimates along the bursts.
\end{abstract}

\section{INTRODUCTION}

Though unnoticed for some time, there has been an increasing interest towards multicarrier and in particular Orthogonal Frequency Division Multiplexing (OFDM), not only for digital audioand video-broadcasting (DAB and DVB) but also for high-speed modems over Digital Subscriber Lines (xDSL), and more recently for small area mobile wireless broadband systems (ETSI BRAN HiperLAN/2, IEEE802.11a and MMAC).

In order to enable a very simple equalization scheme in the frequency domain, classical multicarrier systems insert at the transmitter, after IFFT modulation, a time-domain redundant Cyclic Prefix (CP) of length larger than the FIR channel memory (see e.g., [1]). At the receiver end, $\mathrm{CP}$ is discarded to avoid interblock interference and each truncated block is FFT processed - an operation converting the frequency-selective channel output into parallel flat-faded independent subchannel outputs each corresponding to a different subcarrier. Unless zero, flat fades are removed by dividing each subchannel output with a simple gain equal to the channel transfer function value at the corresponding subcarrier.

Instead of inserting the $\mathrm{CP}$, it was proposed recently in [2] to pad Trailing Zeroes (TZ) (a null signal) at the end of each IFFT modulated block. This new modulation, so termed TZ-OFDM, introduces the same amount of redundancy as CP-OFDM and thus results in the same bitrate loss. Interestingly, TZ-OFDM assures channel-irrespective retrieval of the transmitted symbol blocks even when a channel zero is located on a subcarrier which is not possible with the CP precoder [2]. The price paid by TZ-OFDM is increased receiver complexity (the single FFT required by CP-OFDM is replaced by FIR filtering).

In this paper we take a closer look at TZ-OFDM and establish that even the receiver complexity can be made equal to that of CP-OFDM. Specifically, we propose a simple equalization scheme based on the well-known overlap-and-add (OLA) block convolu- tion algorithm (as opposed to the overlap-and-save (OLS) approach exploited by CP-OFDM). As a trade off, the resulting TZ-OFDMOLA transceiver inherits CP-OFDM's simplicity in equalization but also forsakes TZ-OFDM's channel-irrespective blind identifiability and symbol recovery. Interestingly, based on the OLS/OLA link, we show that in the absence of high-power amplifier (HPA) nonlinearities (NL) TZ-OFDM-OLA is in fact the dual of CP-OFDM system by a transposition of their flow graphs. For this reason, they share the same global transfer function and are expected to perform similarly. Because HPA-NL have to be taken into account in practice, we consider the Peak to Average Power Ratio (PAPR) as figure of merit [3], and compare TZ-OFDM-OLA with CP-OFDM both analytically and with realistic simulations tailored to the practical context of the HiperLAN/2 (HL2) standard.

Additional comparisons are performed between two semi-blind subspace-based channel estimators developed for the CP and TZ precoders of [4] and [5], and both are also compared with the convenional pilot-based approach. To comply with the HL2 standard, some modifications of these algorithms are derived herein in order to account for the presence of zero subcarriers that are used to provide frequency guard-bands between adjacent OFDM systems. Although often ignored in the literature, accounting for these guardbands is important because they cause rank deficiency of the transmitted signal correlation matrix. In addition to modifying subspace channel estimation algorithms, we also show in this paper how to remove their inherent scalar-ambiguity by resorting to a semi-blind least-squares criterion that incorporates pilot subcarriers.

\section{DESCRIPTION OF THE TWO SYSTEMS}

Figure 3 depicts a classical CP-OFDM system. The size $N$ input digital vector $\mathbf{s}(k)$ is first modulated by the inverse of the Fast Fourier Transform (FFT) matrix $\mathbf{F}=\mathbf{F}_{N}^{-1}$. Then a CP of length $D$ is inserted between each generated time domain block vector $\tilde{\mathbf{s}}(k)$. The components of the resulting vector $\tilde{\mathbf{s}}^{\mathrm{cp}}(n)$ are finally sent sequentially through the channel. The total number of time domain samples to be transmitted per block is thus $P=N+D$. The channel effects are modeled by a FIR filter with impulse response coefficients $\mathbf{h}=\left[h_{0} \ldots h_{N-1}\right]^{T}$ and the addition of a white noise $\tilde{b}_{n}$ of variance $\sigma_{b}^{2}$. In practice, the system is designed such that $N \geq D \geq L$ where $L$ is the channel length (i.e. $h_{i>L}=0$ ).

At the receiver, the $\mathrm{CP}$ is simply removed yielding to the frequency domain equivalent parallel carriers model of figure 1 where $\tilde{\mathbf{h}}=\left[H_{0} \ldots H_{N-1}\right]^{T}=\mathbf{F}_{N} \mathbf{h}$, where $H_{k}$ denotes the channel's transfer function on the $k$ th subcarrier. This CP-OFDM property derives from the fast convolution algorithm based on the OLS approach [6] which enables a very simple equalization.

The left part of Figure 4 presents the $\mathrm{TZ}$ precoder as proposed in [2] where the CP insertion is replaced by the transmission of $D$ 
trailing zeroes at the tail of the modulated block $\mathbf{s}(n)$ resulting in $\mathbf{s}^{\mathrm{tz}}(n)$. The right part details the receiver equalization scheme we propose which is based on an OLA approach:

- as for the CP-OFDM case, the linear channel filtering is first turned into circular convolution (modeled by a circulant matrix multiplication) by adding the last $D$ samples (corresponding to the TZ insertion) to the $D$ first ones of the received block;

- because circulant matrices are diagonalized by the FFT [1], just as in the CP case, the channel distortions are viewed after the FFT demodulation as simple scalar gains applied on each subcarrier;

- then the obtained block of samples is simply equalized as usual by multiplying each of its component by an estimate of the corresponding frequency channel coefficient conjugate.

\section{EQUIVALENCES AND COMPARISONS}

Since both $\mathrm{CP}$ and $\mathrm{TZ}$ precoders share a similar simple equalization scheme and give rise to the same parallel flat fading carriers model of figure 1, we are prompted to investigate links between the two transceivers. Towards this objective, one can redraw both systems as MIMO flow graphs [6] and show that TZ-OFDM-OLA with its simple equalization structure is simply the transposition of the CP-OFDM flow graph. In fact, direct application of Tellegen's theorem [6] leads to the following result:

Theorem: Assuming a linear time invariant baseband model (corresponding to a static channel free of non-linearities introduced by the RF front-end), TZ-OFDM-OLA with its simple equalization structure has exactly the same baseband transfer function than the classical OFDM system.

Hence, both systems can be expected to perform the same in a given context. However, it is well known that the HPA introduces nonlinear distortions [3] which destroy orthogonality between the carriers and deteriorate the OFDM system performance by introducing intercarrier interference. Thus, even if correction methods have been developed [7], it is shown below that, for a given clipping ratio (number of clipped symbols divided by the total number of transmitted symbols), the mean transmitted power by TZ-OFDM is smaller compared to CP-OFDM.

In the following, the HPA is modeled as an ideal clipper and the amplitudes of its input, $\left|\tilde{s}_{k}(i)\right|$ are assumed approximately Rayleigh distributed. This assumption is well justified (especially for large $N$ ) since the IFFT precoding that takes place in both CP and TZ precoders maps the finite-alphabet sequence $s_{k}(i)$, to the approximately Gaussian i.i.d. sequence $\tilde{s}_{k}(i)$ that has approximately Rayleigh distributed amplitudes $\left|\tilde{s}_{k}(i)\right|$. Under this assumption we have shown that the clipping ratio of the two transmitters for a given Input BackOff are related as follows:

$$
C_{\mathrm{TZ}}=\frac{N}{P} 10^{-\frac{B_{\text {off }}}{20}} e^{-10^{\frac{B_{\text {off }}}{5}}}=\frac{N}{P} C_{\mathrm{CP}} .
$$

From another angle, if one fixes the clipping ratio to a common value $C=C_{\mathrm{TZ}}=C_{\mathrm{CP}}$, the two precoders require different Input Back-Offs and their transmitter SNR are given by:

$$
S N R_{\mathrm{CP}}=\frac{\sigma_{s}^{2}}{\sigma_{b}^{2}} 10^{-\frac{B_{\text {off }}^{\mathrm{CP}}}{10}}=\frac{P}{N} S N R_{\mathrm{TZ}} 10^{-\frac{B_{\text {off }}^{\mathrm{CP}}-B_{\text {off }}^{\mathrm{TZ}}}{10}}
$$

where $\sigma_{s}^{2}\left(\sigma_{b}^{2}\right)$ denotes the information symbol (noise) variance. The SNR difference $\Delta_{\mathrm{SNR}}:=S N R_{\mathrm{CP}}-S N R_{\mathrm{TZ}}$ can be found from
(2) as: $\Delta_{S N R}=10 \log \left(1+\frac{D}{N}\right)+B_{\text {off }}^{\mathrm{TZ}}-B_{\text {off }}^{\mathrm{CP}} \approx 10 \log \left(1+\frac{D}{N}\right)$ where the last approximation holds for small clipping ratios. For the HL2 transmissions detailed in the next section, $D / N=0.25$, and figure 2 shows that clipping effects alone entail excess SNR incurred by the $\mathrm{CP}$ relative to that required by $\mathrm{TZ}$ precoding as high as $0.96 \mathrm{~dB}$. However, this degradation may be compensated by some particular properties of the TZ precoder such as the existence of the specific subspace channel estimation method presented in 5. This motivates our subsequent comparison between the TZ-OFDM-OLA and the CP-OFDM systems based on simulations for a specific scenario which also incorporates different channel estimation methods.

\section{HIPERLAN/2 AND CSI WITH TRAINING}

We have chosen to compare the two precoders in the practical context of the HL2 broadband wireless communication standard (similar to IEEE802.11a) currently under definition. HL2 is a multicarrier systems operating over $20 \mathrm{MHz}$ in the $5 \mathrm{GHz}$ band at typical SNR values of 5-10 dB for terminal speeds $v \leq 3 \mathrm{~m} / \mathrm{s}$. The number of carriers is $N=64$ and the $\mathrm{CP}$ is $D=16$ samples long. Among these $N$ carriers, 12 carriers are null-carriers (including the middle null corresponding to the DC component and the zeros padded on both ends $\mathrm{cf}$. figure 3 in order to provide frequency guard bands against co-channel interference from adjacent OFDM systems). Among the remaining $K=52$ "central" subcarriers, 4 are fixed pilots carrying known QPSK symbols $P_{1}-P_{4}$ while the rest $U=K-4=48$ subcarriers convey the information bearing sequence.

With $X$ denoting each of the 48 information symbols drawn from 4, 16, or, 64-QAM constellations (depending on the target bit rate), the corresponding frequency domain OFDM symbol structure is summarized below:

$|\underbrace{0 \cdots 0}_{6} \underbrace{X \cdots X}_{5} P_{1} \underbrace{X \cdots X}_{13} P_{2} \underbrace{X \cdots X}_{6} 0 \underbrace{X \cdots X}_{6} P_{3} \underbrace{X \cdots X}_{13} P_{4} \underbrace{X \cdots X}_{5} \underbrace{X \cdots 0}_{5}|$

The first two blocks of the burst $(\mathbf{s}(0)$ and $\mathbf{s}(1))$ contain training symbols which are known to the receiver and can be used for channel status information (CSI) acquisition. Relying on these known training symbols and the received noisy FFT processed data $r_{i}(k)$, the OFDM receiver forms an initial channel estimate using:

$$
\widehat{H}_{i}(1)=\frac{1}{2}\left[r_{i}(0) / s_{i}(0)+r_{i}(1) / s_{i}(1)\right] \text { for } 0 \leq i<N
$$

This method is the classical pilot-based estimation algorithm usually adopted by coherent modulation OFDM schemes [8].

Because only the entries $m=12,26,40,54$ contain known (pilot) symbols in subsequent blocks $\mathbf{s}(k)_{k \geq 2}$, one can track adaptively the channel transfer function using a running average (over say $B=10$ blocks) based only on these 4 frequencies as follows:

$$
\widehat{H}_{i}(k+1)=\frac{1}{B} \sum_{l=0}^{B-1} r_{i}(k-l) / s_{i}^{\mathrm{pil}}(k-l) \text { for } i \in\{12,26,40,54\}
$$

Actually the standard specifies these 4 pilot carriers for synchronization and phase tracking purposes (in a coherent modulation scheme) but they are too distant in frequency (spaced more than the channel coherence bandwidth) for estimating the channel by a simple interpolation or even for tracking channel variations.

Thus only a partial channel tracking can be achieved using (??) which may not yield accurate channel estimation in rapidly varying environments. To enhance mobility in HL2, semi-blind channel estimation is well motivated especially with the relatively small 
number of carriers that enable even subspace approaches to be tried with affordable complexity.

\section{SUBSPACE-BASED CHANNEL ESTIMATION}

In the following only the TZ precoder is considered. The corresponding equations for the $\mathrm{CP}$ precoder have been skipped for the sake of conciseness but can be straightforwardly derived from [4].

Consider the system depicted in figure 4 and define $\overline{\mathbf{H}}_{0}$ the $P \times N$ as the lower triangular Toeplitz matrix with first column $\left[h_{0}, \ldots, h_{D}, 0, \ldots, 0\right]^{T}$. The expression of the received block symbol is given by: $\tilde{\mathbf{s}}^{\mathrm{tz}}(k)=\overline{\mathbf{H}}_{0} F \mathbf{s}(k)+\tilde{\mathbf{b}}(k)$. Be $R:=E\left[\tilde{\mathbf{s}}^{\mathrm{tz}}(k) \tilde{\mathbf{s}}^{\mathrm{tz}}(k)^{H}\right]$ the received block symbol autocorrelation matrix. It is obvious that $\operatorname{rank}\left(\overline{\mathbf{H}}_{0}\right)=N$ unless $\mathbf{h}=\mathbf{0}$ and therefore the noise subspace of $R$ has rank $D$. Denoting by $\mathbf{G}=\left[\mathbf{g}_{1}, \ldots, \mathbf{g}_{D}\right]$ a basis of this noise subspace, it can be shown that $\mathbf{h}$ can be uniquely identified solving the linear equations system $\mathbf{G}^{H} \overline{\mathbf{H}}_{0}=0$.

Finally this system can be rewritten as: $\mathbf{h}^{H} \mathcal{G}=0$ by noticing that $\mathbf{g}_{i}^{H} \overline{\mathbf{H}}_{0}=\left[g_{i}(0), \ldots, g_{i}(P-1)\right]^{*} \overline{\mathbf{H}}_{0}=\mathbf{h} \mathcal{G}_{i}^{*}$ where $\mathcal{G}_{i}$ is the $D \times N$ Hankel matrix with first column $\left[g_{i}(0), \ldots, g_{i}(D)\right]^{T}$ and with last row $\left[g_{i}(D), \ldots, g_{i}(P-1)\right]$. In practice $R$ is only estimated by an averaging in time: $\widehat{R}_{\tilde{\mathbf{r}} \tilde{\mathbf{r}}}^{(M)}=\frac{1}{M} \sum_{k=0}^{M-1} \tilde{\mathbf{r}}(k) \tilde{\mathbf{r}}(k)^{H}$ and therefore $\mathbf{G}^{H} \tilde{\mathbf{H}}_{0}=0$ has to be solved in the least square sense leading to the following quadratic optimization criterion:

$$
\widehat{\mathbf{h}}=\operatorname{argmin}\left(\sum_{i=1}^{D}\left\|\widehat{\mathcal{G}}_{i}^{H} \mathbf{h}\right\|^{2}\right)=\operatorname{argmin} \mathbf{h}^{H}\left(\sum_{i=1}^{D} \widehat{\mathcal{G}}_{i} \widehat{\mathcal{G}}_{i}^{H}\right) \mathbf{h}
$$

The presence of null side carriers is not specific to HL2 but is a common feature of all current standardized OFDM systems (DAB, DVB, etc.) These virtual carriers entails that the autocorrelation matrix $R_{\mathrm{ss}}$ has now a rank $K$ instead of $N$. This generates some problems with the subspace algorithms that cannot be applied directly and have to be modified. The required adjustments are detailed below.

The received signal $\tilde{\mathbf{r}}^{\mathrm{tz}}(k)$ can now be expressed as: $\tilde{\mathbf{r}}^{\mathrm{tz}}(k)=$ $\overline{\mathbf{H}}_{0} \mathbf{F}_{\text {tr }} \mathbf{s}_{\mathrm{tr}}(k)$ instead of $\tilde{\mathbf{r}}^{\mathrm{tz}}(k)=\overline{\mathbf{H}}_{0} \mathbf{F} \mathbf{s}(k)$ where $\mathbf{F}_{\text {tr }}$ is the truncated $N \times K$ matrix obtained from $\mathbf{F}$ by removing the columns corresponding to the zero entries of $\mathbf{s}(k)$ and where $\mathbf{s}_{\mathrm{tr}}(k)$ corresponds to the non-zero components of $\mathbf{s}(k)$.

Thus, in that case, the channel estimation is obtained considering equation: $\mathbf{G}^{H} \overline{\mathbf{H}}_{0} \mathbf{F}_{\text {tr }}=\mathbf{0}$ instead of $\mathbf{G}^{H} \overline{\mathbf{H}}_{0}=\mathbf{0}$. Note that this equation leads to $\mathbf{h}^{H} G \mathbf{F}_{\mathrm{tr}}=\mathbf{0}$ by commuting the vector-matrix product as detailed in 5 .

In [4], a semi-blind algorithm has been proposed which directly applies to the present context. This algorithm accelerates the convergence of the blind algorithm by taking benefit of the training sequence sent at the beginning of each burst to initialize the autocorrelation matrix estimation. However it does not remove the inherent blind method scalar indetermination. Indeed the channel is identified by minimizing (??) subject to a properly chosen constraint for avoiding the trivial zero solution. This leads to a channel estimation up to a scalar coefficient $\alpha$ : $\widehat{\mathbf{h}}^{\text {sub }}=\alpha \widehat{\mathbf{h}}$ and $\alpha$ can be inferred from the pilot carriers as described below.

Denote by $r_{1}^{\text {pil }}, \ldots, r_{4}^{\text {pil }}$ the symbols received on the pilot carriers. An estimation of the channel attenuations at the corresponding frequencies is provided by:

$$
\widehat{\mathbf{H}}^{\mathrm{pil}}=\left[r_{1}^{\mathrm{pil}} / P_{1}, \ldots, r_{4}^{\mathrm{pil}} / P_{4}\right]^{T}
$$

Another estimation of these coefficients can be inferred from the the subspace identification (up to $\alpha$ ):

$$
\widehat{\mathbf{H}}^{\text {sub }}=\mathbf{F}^{\mathrm{pil}} \widehat{\mathbf{h}}^{\mathrm{sub}}=\alpha \mathbf{F}^{\mathrm{pil}} \widehat{\mathbf{h}}=\alpha \widehat{\mathbf{H}}
$$

where $\mathbf{F}^{\text {pil }}$ is the matrix obtained from the size $N$ FFT matrix $\mathbf{F}_{N}$ by selecting the pilot carriers rows and by removing the others. Thus, coupling (??) and (??), $\alpha$ can be determined by solving the linear system $\widehat{\mathbf{H}}^{\text {sub }}=\widehat{\alpha} \widehat{\mathbf{H}}^{\text {pil }}$ in the least square sense. However if the channel estimation $\widehat{\mathbf{h}}^{\text {sub }}=\alpha \widehat{\mathbf{h}}$ obtained using the subspace algorithm is far from the true CIR $\mathbf{h}$ (up to $\alpha$ ), the final channel estimation will remain inaccurate even if $\alpha$ is estimated such that $\left\|\mathbf{h}-\widehat{\mathbf{h}}^{\text {sub }} / \alpha\right\|$ is minimal. Somehow no benefit is taken from from the knowledge of the channel attenuations on the pilot carriers for the subspace algorithm. This can be achieved by considering the composite following modified linear system including a second member:

$$
\left\{\begin{aligned}
\mathcal{G}_{i}^{H} \mathbf{h} & =0 \text { for } 1 \leq i \leq D \\
\mathbf{F}^{\text {pil }} \mathbf{h} & =\mathbf{H}^{\text {pil }}
\end{aligned}\right.
$$

Since this equation is only approximatively verified in practice, it has to be solved in the least square sense similarly to (??) which leads to the minimization of the following criterion:

$$
\widehat{\mathbf{h}}=\operatorname{argmin}\left(\sum_{i=0}^{D-1}\left\|\widehat{\mathcal{G}}_{i}^{H} \mathbf{h}\right\|^{2}+\left\|\mathbf{F}^{\mathrm{pil}} \mathbf{h}-\widehat{\mathbf{H}}^{\mathrm{pil}}\right\|^{2}\right)
$$

This criterion, whose solution is given by $\widehat{\mathbf{h}}=\left(\mathbf{Q}^{\text {pil }}\right)^{-1} \mathbf{F}^{\text {pil }}{ }^{H} \widehat{\mathbf{H}}^{\text {pil }}$ where $\mathbf{Q}^{\text {pil }}=\sum_{i=0}^{D-1} \widehat{\mathcal{G}}_{i} \widehat{\mathcal{G}}_{i}^{H}+\mathbf{F}^{\text {pil }}{ }^{H} \mathbf{F}^{\text {pil }}$, is very close to the semiblind criterion already proposed for another context in [9]. The difference in our case is that the training symbols cannot provide alone a channel estimation.

As illustrated in the simulations, the resulting semi-blind algorithm combining all the previous described enhancements outperforms the classical pilot-based channel estimation.

\section{SIMULATIONS}

This section presents a comparison of the CP-OFDM and the TZ-OFDM-OLA schemes using either the classical pilot-based or subspace channel identification algorithm. All the results have been obtained running Monte Carlo simulations, each trial corresponding to a different realization of the typical $5 \mathrm{GHz}$ wireless indoor channel A model specified by HL2. Note that for enabling a fair comparison between the various algorithms an extra denoising of the estimation is applied to the classical pilot based method. This refinement consists in taking into account in the estimation process that channel in the frequency domain actually corresponds in the time domain to a FIR of length $\approx N / 4$ [10].

The channel estimation Mean Square Error (MSE) is only relevant on the $U$ useful carriers since only these subbands will be equalized and is defined as: $M S E=\frac{1}{U} \sum_{u \in \mathcal{U}}|H(u)-\widehat{H}(u)|^{2}$ where $\mathcal{U}$ is the set of indices corresponding to the $U$ useful carriers.

Moreover, in order to better quantify the impact of the channel estimation on the system overall performance, we use the following "effective SNR" criterion [11] defined as:

$$
S N R_{\text {eff }}=-10 \log _{10}\left(10^{-S N R / 10}+10^{M S E / 10}\right)
$$

This criterion can indeed be interpreted as the comprehensive SNR observed at the receiver by the Viterbi decoder. In fact, the Viterbi algorithm decodes the transmitted symbols $s_{i}$ on the carrier $i$, having an estimate $\widehat{H}_{i}$ of $H_{i}$ by minimizing the metric $m_{i}=\left|r_{i}-\widehat{H}_{i} s_{i}\right|^{2}=$ 
$\left|r_{i}-H_{i} s_{i}-\Delta\left(H_{i}\right) s_{i}\right|^{2}$. Hence since practical OFDM systems always implement frequency interleaving, the perturbation $\Delta\left(H_{i}\right) s_{i}$ can be approximated by a white Gaussian noise. Therefore it can be gathered with the classical thermal noise into a single noise term which justifies equation (??). Thus the effective SNR jointly takes into account the real noise and the channel estimation error and is a reliable criterion of the system performance. However, it is important to notice that this criterion only makes sense with the CP-OFDM and TZ-OFDM-OLA equalization schemes and is not relevant for extrapolating coded BER in another context (e.g. the robust original TZ-OFDM transceiver proposed in [2]).

Figure 5 illustrates the evolution of the channel estimation MSE along the burst assumed here to contain 500 OFDM symbols. It clearly appears that the channel estimations provided by the classical pilot-based method degrade quickly when the channel is varying whereas the subspace algorithms track approximatively its variations. The channel estimation turns to be more accurate for the TZ algorithm after 150 symbols (about $0.75 \mathrm{~dB}$ at the end of the frame) which already corresponds to very long bursts. This can be explained by the fact that the size of the autocorrelation matrix used by the TZ subspace algorithm is half the size of the one processed by the $\mathrm{CP}$ one.

Figure 6 displays the effective SNR averaged over the burst as a function of the thermal SNR. This figure is the most meaningful since it provides a more reliable idea of the system mean BER performance than the channel MSE. When using the subspace algorithms compared to the classical method, a gain of about $0.5 \mathrm{~dB}$ at $\mathrm{SNR}=5 \mathrm{~dB}$ and $1.5 \mathrm{~dB}$ at $\mathrm{SNR}=10 \mathrm{~dB}$ can be expected. Actually the difference in performance between the $\mathrm{CP}$ and the $\mathrm{TZ}$ transceivers is tiny (between 0 and $0.25 \mathrm{~dB}$ ) and could be further reduced when dealing with smaller bursts.

\section{CONCLUSIONS}

This paper has presented a simple equalization scheme for the TZ precoder and a subspace channel estimation algorithm. A comparison between the TZ-OFDM-OLA and CP-OFDM transceivers has been conducted in the HL2 context. As expected because of the duality of the two systems, the two schemes exhibit very similar performance. Accounting for the nonlinearities introduced by the clipper and PAPR issues, it has been illustrated that the CP precoder requires a smaller input power back-off than the TZ leading to a larger SNR of operation. This results in a better performance for CP-OFDM when transmitting small bursts. However, with long bursts TZ-OFDM-OLA is to be preferred because it has better channel tracking capabilities than CP-OFDM. Moreover, one may also benefit by using the more complex TZ-OFDM receiver that has the capability to retrieve the information sent on a carrier corresponding to a channel zero which is not the case for the CP-OFDM or the TZ-OFDM-OLA system considered herein. Note that this could bring significant performance gains. Finally, this paper has highlighted that semi-blind methods could be considered as a practical solution for tracking channel variations and improving the pilotbased channel estimation accuracy.

\section{REFERENCES}

[1] A. Akansu, P. Duhamel, X. Lin, and M. de Courville. Orthogonal Transmultiplexers in Communication : A Review. IEEE Trans. on Signal Processing, 463(4):979-995, April 1998.

[2] A. Scaglione, G.B. Giannakis, and S. Barbarossa. Redundant Filterbank Precoders and Equalizers - Part I : Unification and Optimal Designs. IEEE Trans. on Signal Processing, 47:1988-2006, July 1999.
[3] R. van Nee and A. de Wilde. Reducing the Peak to Average Power Ratio of OFDM. In Proceedings of the Vehicular Technology Conference, pages 2072-2076, 1998.

[4] B. Muquet, M. de Courville, P. Duhamel, and V. Buzenac. A Subspace based blind and semi-blind channel identification method for OFDM systems. In Proc.of SPAWC'99, pages 170-173, May 1999.

[5] A. Scaglione, G.B. Giannakis, and S. Barbarossa. Redundant Filterbank Precoders and Equalizers - Part II : Blind Channel Estimation, Synchronization and Direct Equalization. IEEE Trans. on Signal Processing, 47:2007-2022, July 1999.

[6] R. Crochiere and L. Rabine. Multirate Digital Signal Processing. In Multirate Digital Signal Processing. Prentice-Hall, Englewood Cliffs, New Jersey, USA, 1983.

[7] A.L. Kambanellas and G.B. Giannakis. Modulo Pre-Equalization of Nonlinear Communication Channels. In Proceedings of the IEEE Workshop on Signal Processing Advances for Wireless Communications, Annapolis, USA, May 1999.

[8] V. Mignone and A. Morello. CD3-OFDM: A Novel Demodulation Scheme for Fixed and Mobile Receivers. IEEE Trans. on Communications, 44(9):1144-1151, September 1996.

[9] A. Gorokhov and P. Loubaton. Semi-blind second order identification of convolutive channels. In IEEE International Conference on Acoustics, Speech, and Signal Processing, Munich, Germany, April 1997.

[10] J. van de Beek, O. Edfors, M. Sandell, S. Wilson, and P. Borjesson. On Channel Estimation in OFDM Systems. In Proceedings of the Vehicular Technology Conference, volume 2, pages 815-819, Chicago, USA, July 1995.

[11] M. Speth and H. Meyr. Complexity constrained channel estimation for OFDM based transmission over fast fading channels. In Proceeding of the European Personal Mobile Communications Conference, Paris, France, March 1999.
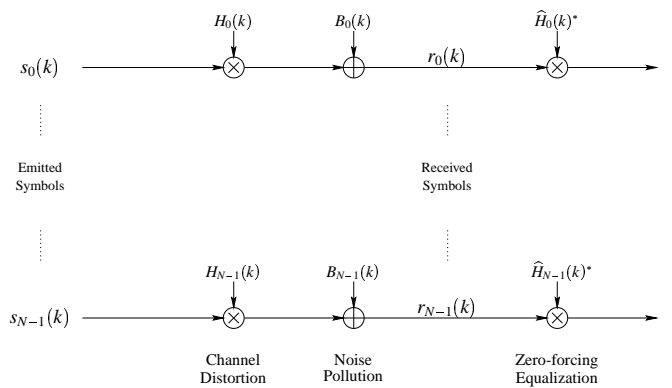

Figure 1: Equivalent parallel flat fading carriers model

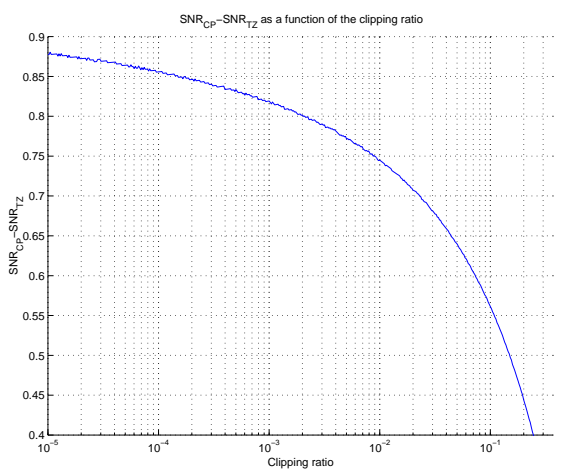

Figure 2: SNR difference between the two precoders induced by clipping effects alone 


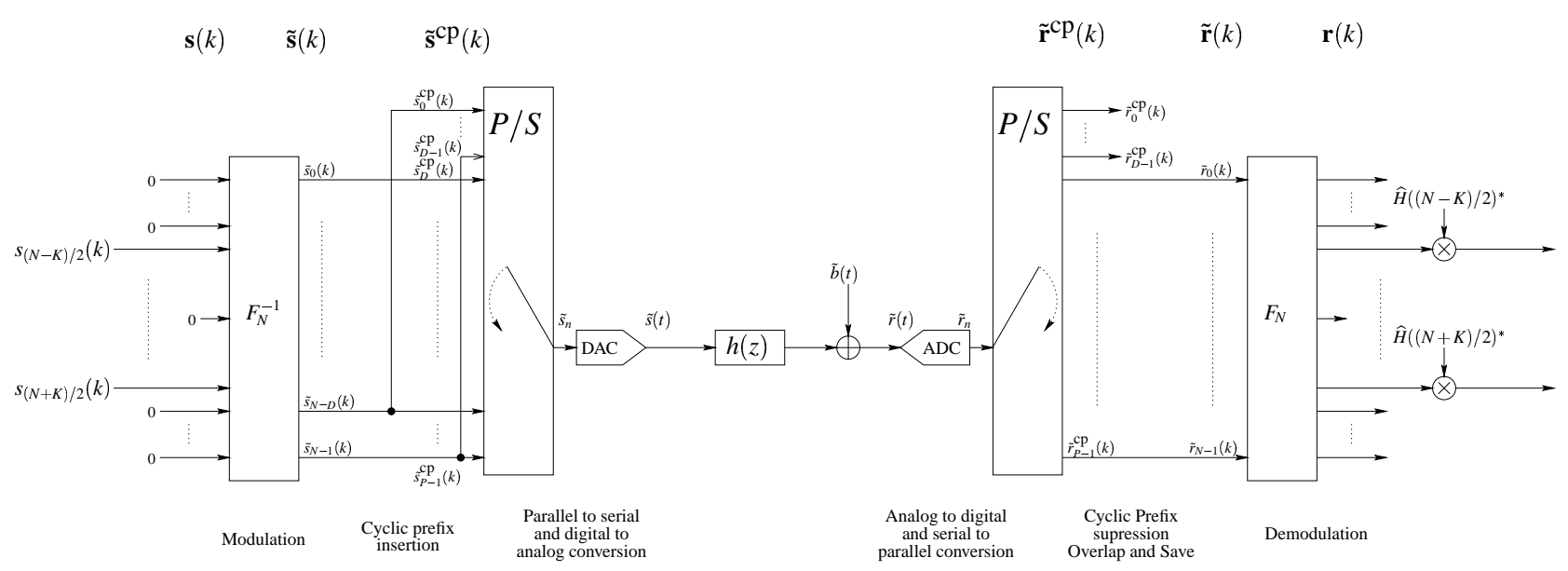

Figure 3: Parallel Carriers CP transceiver

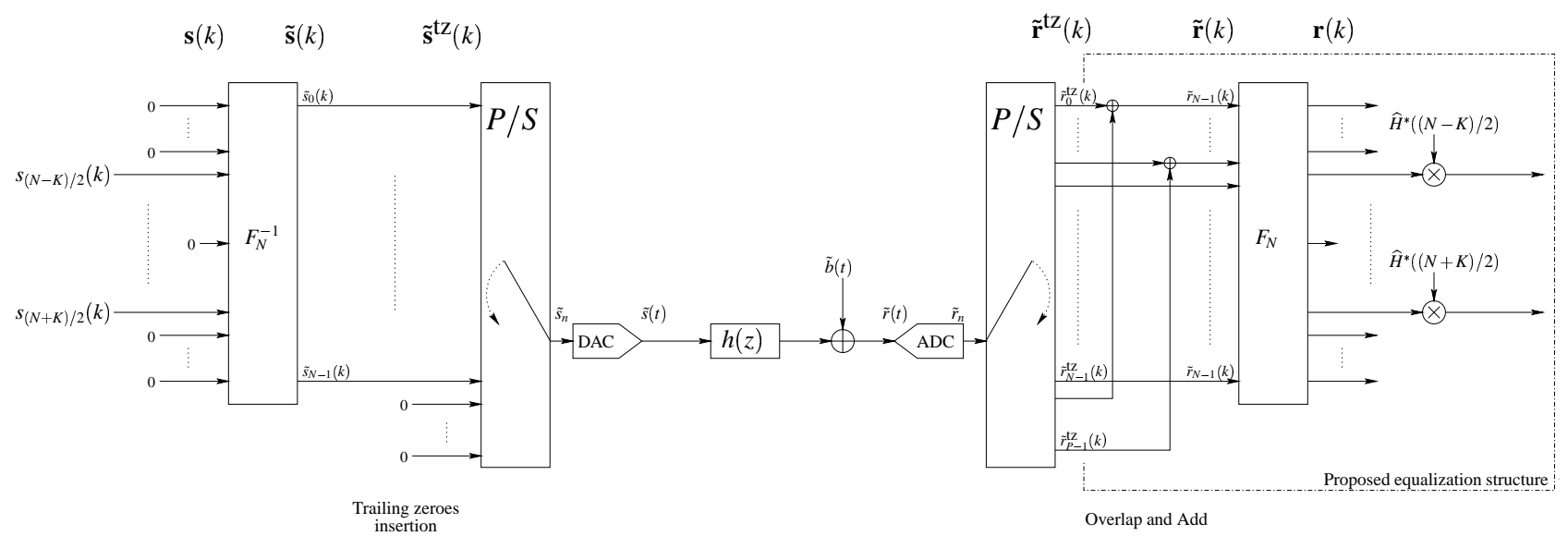

Figure 4: Parallel Carriers TZ transceiver

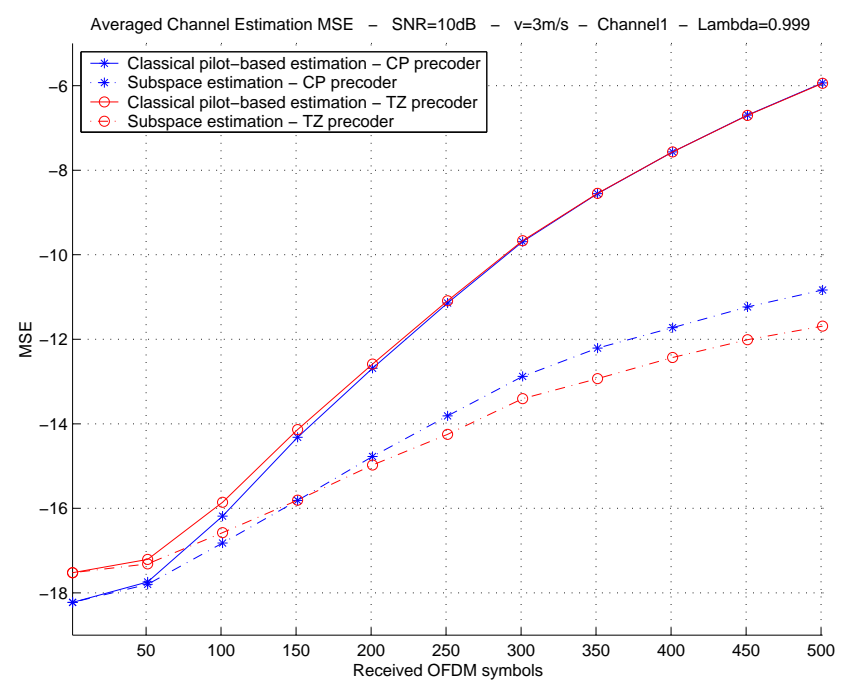

Figure 5: MSE along the frame at $\mathrm{SNR}=10 \mathrm{~dB}, v=3 \mathrm{~m} / \mathrm{s}$

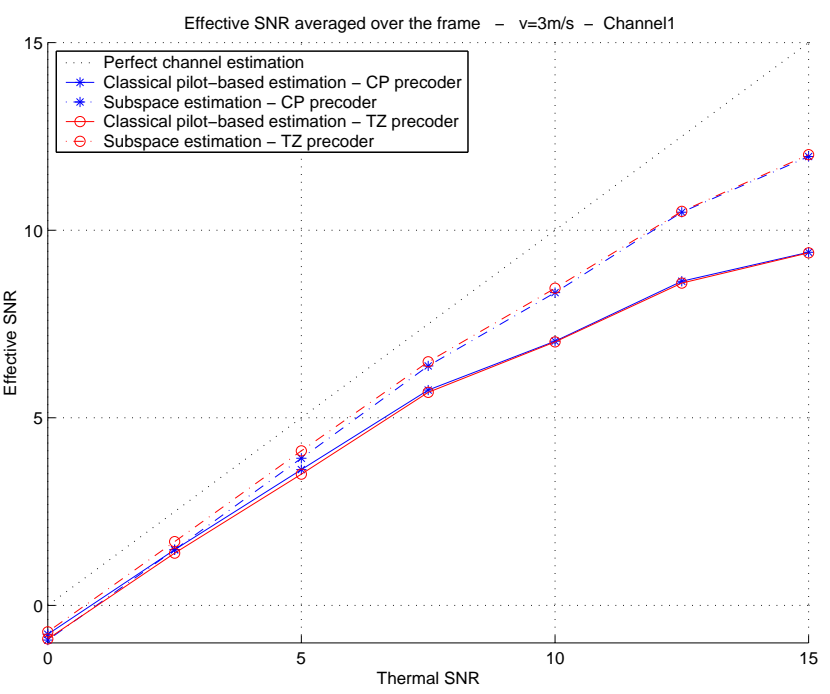

Figure 6: Effective SNR averaged over the frame 